\title{
Quantitative Assessment of the Infarct Transmurality Using Delayed Contrast Enhanced Magnetic Resonance Images: Description and Validation
}

\author{
N Kachenoura ${ }^{1}$, A Redheuil ${ }^{1,2}$, R El-Berbari ${ }^{1}$, C Ruiz Dominguez ${ }^{1}$, \\ A Herment ${ }^{1}$, E Mousseaux ${ }^{1,2}$, F Frouin $^{1}$ \\ ${ }^{1}$ INSERM, U678, Laboratory of functional imaging, Paris, France \\ ${ }^{2}$ Cardiovascular Radiology Department, HEGP, Paris, France
}

\begin{abstract}
The extent and degree of myocardial injury after an ischemic event are strong predictors of patient's outcome. After acute infarction, delayed contrast enhancement magnetic resonance imaging allows clinicians to distinguish between viable and non-viable myocardium and can delineate with high precision the infarcted tissue. The aim of this study is to provide a quantitative method based on the fuzzy c-means clustering algorithm to assess the location and the extent of the infarcted area. Segmental infarct trasmurality was visually assessed on a 5-point scale for the 288 segments. The agreement between visual and quantitative analyses was good: $84 \%$ of the segments were categorized similarly by quantitative and visual analyses.
\end{abstract}

\section{Introduction}

Noninvasive assessment of myocardial infarct size is important in the follow-up of patients with coronary artery disease because of its known prognostic value (1). After myocardial infarction it allows the evaluation of myocardial viability, which has an important impact in patient management. One of the main challenges remains to differentiate reversible from nonreversible myocardial injury.

Several methods have been developed for the measurement of infarct size, such as contrast echocardiography (2) which, however, is not able to delineate specifically nonreversible injury. Another method validated and accepted in clinical routine is based on nuclear imaging techniques; it provides semi quantification of the infarct size (3).

Several studies show the relevance of magnetic resonance imaging in the assessment of myocardial viability which can be assessed using contraction MR studies under pharmacological stress (4) or more directly thanks to delayed enhancement images which illustrate with high accuracy even small infarcts (5). Thanks to its precision, this new method has proven its superiority over nuclear methods particularly in the detection of sub endocardial necrosis (6). The delayed enhancement image is acquired 10 to 20 minutes after the injection of the contrast agent. At this steady-state, non viable regions are characterized by hyper-enhanced signal (7). Myocardial functional recovery after an acute coronary event is inversely correlated with the extent of non viable myocardium (8). Therefore, it is crucial to measure precisely and with high reproducibility infarct transmurality.

In clinical routine, delayed enhancement images are interpreted visually (9). To reduce the subjectivity of visual assessment, a number of quantitative approaches have been developed. The first methods use thresholds which are based on statistical criteria such as the mean gray level and its standard deviation in the myocardium. However, the threshold value depends on the study and can vary from 1 to $6 * \mathrm{SD}(7,10,11)$. The second semiautomatic approach defines a threshold value as a mean value of two regions, the first one being delineated in the remote myocardium and the second in the infracted tissue. This threshold combined with the gray level on the centerline chords provides the infarct transmurality. Finally a method based on full-width at half maximum (FWHM) criteria was developed, the user clicks in the hyperenhanced region, and a multi pass growing algorithm is used to delineate the infarcted area (12). For all these methods, in addition to the delineation of myocardial borders, the operator has to delineate a region within a remote and or infarcted tissue which increases variability (13).

In this paper, a semi-automatic method based on the unsupervised algorithm of the fuzzy c-means clustering was developed to quantify myocardial infarct extent and to provide segmental delayed enhancement scores.

\section{Methods}

\subsection{Image acquisition and preprocessing step}


MR acquisition for 15 patients having myocardial infarction and 3 normal subjects were acquired at European Hospital Georges Pompidou (HEGP) according to a standard clinical protocol. Images were acquired using a clinical GEMS Signa CV/i ${ }^{\mathrm{TM}} 1.5 \mathrm{~T}$ magnet. For each patient, three short axis slices were selected (apical, mid-ventricular and basal). Myocardial borders were outlined manually by an expert. Then myocardial segments were defined using two manually placed points (the centre of the left ventricle and the superior intersection between the two ventricles) according to the recommended multimodality 17-segments model (14). Segmental infarct size was visually assessed by an expert on a 5-point scale:

0: No infarction,

1: Transmurality $\leq 25 \%$,

2: Transmurality $26 \%$ to $50 \%$,

3: Transmurality $51 \%$ to $75 \%$,

4: Transmurality $76 \%$ to $100 \%$.

\subsection{Image processing}

Inside the region delimited by the epicardial contour, including the myocardium and the cavity, the fuzzy kmeans algorithm (number of clusters $=2$, parameter of fuzziness =2) was applied. Two classes were estimated: the first class referred to us (E) contains enhanced pixels; it is represented by both the cavity and the infarcted tissue. The second class contains none enhanced pixels (NE). For each pixel $p$, the membership probability to the class $\mathrm{E} u(p, E)$ is stored.

These probabilities are restricted to the myocardium. Each segment is then divided into 4 layers using successively the centerline method (Figure 1). These layers are called $\mathrm{L}_{1}, \mathrm{~L}_{2}, \mathrm{~L}_{3}, \mathrm{~L}_{4}$ from the endocardial border to the epicardial border.

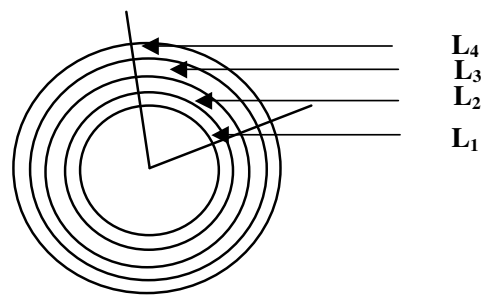

Figure 1: Four myocardial layers

Thereafter, each segment is subdivided in three sub segments, resulting in 48 sub segments per patient (12 sub segments for the apical slice and 18 for both midventricular and basal slices).

For each sub segment $S_{j}(j=1: 48)$ and for each layer $L_{k}$ $(\mathrm{k}=1: 4)$ a mean membership probability $\mathrm{U}(\mathrm{j}, \mathrm{k})$ is computed and called enhancement index :

$$
U(j, k)=\operatorname{mean}_{p \in\left(S_{j} \cap L_{k}\right)}(u(p, E))
$$

\subsection{Segmental scoring}

For each patient enhancement indexes $\mathrm{U}(\mathrm{j}, \mathrm{k})(\mathrm{j}=1$ to $48, \mathrm{k}=1$ to 4 ) are stored and then a threshold value (TH) is used to give for each sub segment a transmurality extent according to a decision tree (Figure 2). This decision tree takes into account the fact that, within the framework of the myocardial infarction, epicardial attack can't occur without endocardial attack.

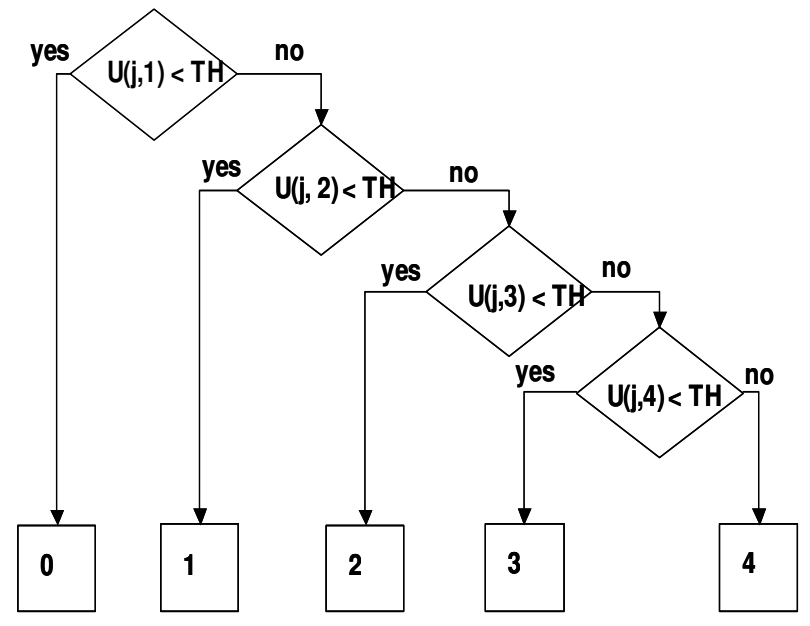

Figure 2: scoring process

For each standard segment three scores are allocated. To take the final decision we use the following analysis:

- If the three sub segments have the same score this one defines the global score of the segment.

- If the three sub segments have three different scores then the highest score defines the global score of the segment.

- If two sub segments present the same score and the third sub segment a different score then there are two possibilities:

- 1) if the difference between the two scores is equal to one then the score of the two sub segments defines the global score of the segment.

- 2) if the difference between the two scores is higher than one the highest score defines the global score of the segment.

The last step of this analysis has two main targets: the first one is to avoid artifacts by ignoring slight and isolated differences. The second target is to design a pessimistic method, which is important to avoid serious 
errors when this method is used to help in diagnosis.

\section{Results}

Classifying on both cavity and myocardium is useful to locate accurately myocardial infarct, but also to affect to the healthy myocardium low membership probabilities to the E class (figure 3 ).

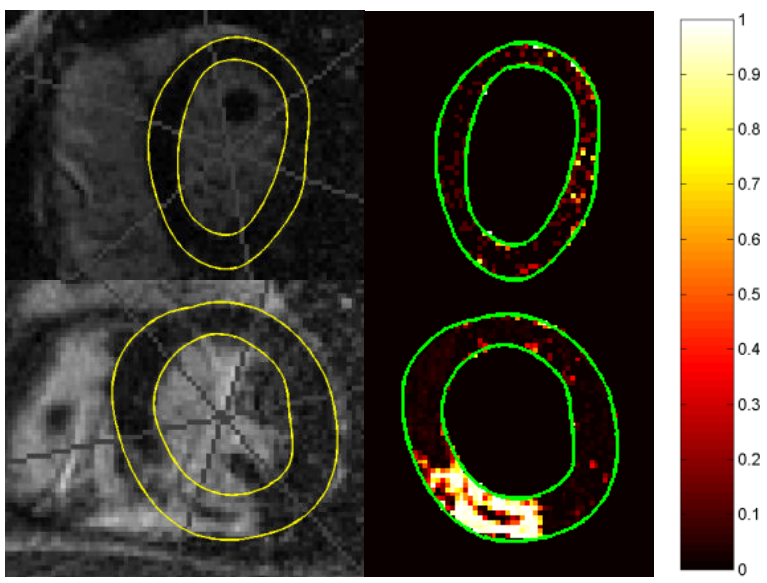

Figure 3: maps of the membership probability for the class (E) on a healthy subject (first line) and on infarcted myocardium (second line).

The segments of the database were classified thanks to a chosen threshold value $(\mathrm{TH}=37)$. A variation of $10 \%$ around $\mathrm{TH}$ has no serious effect on the final diagnosis.

Thus, a head-to-head comparison was performed between the visual classification, done by the expert and the semi-automatic analysis.

Visual assessment

Fuzzy c-means

\begin{tabular}{|c|c|c|c|c|c|}
\hline & 0 & 1 & 2 & 3 & 4 \\
\hline 0 & 20 & & & & \\
\hline & 7 & 3 & 1 & & \\
\hline 1 & 8 & 5 & 4 & & \\
\hline 2 & & 9 & 7 & 6 & \\
\hline 3 & & & 1 & 8 & 3 \\
\hline 4 & 1 & & 2 & 8 & 16 \\
\hline
\end{tabular}

Tab.1: Contingency table

Global agreement shows $84 \%$ of segments categorized similarly by visual and quantitative analyses.

\section{Discussion and conclusion}

In this paper a semi-automatic method for quantifying the infarct extent on delayed enhancement images is proposed. It shows the relevance of using the fuzzy cmeans algorithm.

This approach has some limits: first of all, contours of the myocardium were traced manually. This operation is time consuming and it also introduces variability. Indeed, if the endocardial contour particularly is badly placed, pixels belonging to the cavity risk to be placed in the myocardium and to be classified like delayed enhancement because of their strong intensity.

Taking into account the difficulty of positioning endocardial contour on the delayed enhancement images (because of low contrast between the cavity and the infarcted myocardium), the use of the contraction data may help in contour tracing and increase its quality.

Secondly, the developed method is sensitive to the artefacts in the image ( 1 segment was scored 0 by the expert and 4 by the quantitative method).

In addition, $10 \%$ of the segments are composite (part pathological part healthy myocardium): this problem is inherent to the 17 segment model. These segments were taken into account in the developed method by dividing each segment into 3 sub segments. This operation improves the classification of all composite segments except for three segments. Two of them have a part which is scored 4 while the other part is scored less than 4 . Given that the developed method is pessimistic (Tab1) these segments are scored 4 while scored 2 by the expert. The last segment has a very small located infarct scored 2 by the expert and 0 by our method. To have a better classification for this segment, we have to divide it into 4 sub segments. In this case the number of pixels within each region will decrease and the effect of noisy pixels would increase. In spite of the problems inherent in acquisitions and in myocardial contours tracing, the proposed method provides very encouraging results. However, the evaluation must be extended to a larger number of patients, to show its value versus a strictly visual approach.

In this study, the goal was to estimate the transmural extent of myocardial infarcts on 2D images but the developed method can also provide infarct size. Its application to 3D data would allow estimating infarct volume which is a strong prognostic factor for the patient and a valuable tool in patient medical management.

\section{References}

[1] Gersh BJ, Anderson JL. Thrombolysis and myocardial salvage. Results of clinical trials and the animal paradigm-paradoxic or predictable? Circulation 1993;88(1):296-306.

[2] Kaul S. Assessing the myocardium after attempted reperfusion: should we bother? Circulation 
1998;98(7):625-7.

[3] Gibbons RJ, Miller TD, Christian TF. Infarct size measured by single photon emission computed tomographic imaging with $(99 \mathrm{~m}) \mathrm{Tc}$-sestamibi: A measure of the efficacy of therapy in acute myocardial infarction. Circulation 2000;101(1):101-8.

[4] Kim RJ, Hillenbrand HB, Judd RM. Evaluation of myocardial viability by MRI. Herz 2000;25(4):417-30.

[5] Wagner A, Mahrholdt H, Holly TA, Elliott MD, Regenfus M, Parker M, et al. Contrast-enhanced MRI and routine single photon emission computed tomography (SPECT) perfusion imaging for detection of subendocardial myocardial infarcts: an imaging study. Lancet 2003;361(9355):374-9.

[6] Kuhl HP, Beek AM, van der Weerdt AP, Hofman MB, Visser CA, Lammertsma AA, et al. Myocardial viability in chronic ischemic heart disease: comparison of contrastenhanced magnetic resonance imaging with (18)Ffluorodeoxyglucose positron emission tomography. J Am Coll Cardiol 2003;41(8):1341-8.

[7] Gerber BL, Garot J, Bluemke DA, Wu KC, Lima JA. Accuracy of contrast-enhanced magnetic resonance imaging in predicting improvement of regional myocardial function in patients after acute myocardial infarction. Circulation 2002;106(9):1083-9.

[8] Kim RJ, Wu E, Rafael A, Chen EL, Parker MA, Simonetti $\mathrm{O}$, et al. The use of contrast-enhanced magnetic resonance imaging to identify reversible myocardial dysfunction. N Engl J Med 2000;343(20):1445-53.

[9] Wu E, Judd RM, Vargas JD, Klocke FJ, Bonow RO, Kim RJ. Visualisation of presence, location, and transmural extent of healed Q-wave and non-Q-wave myocardial infarction. Lancet 2001;357(9249):21-8.
[10] Kuhl HP, Papavasiliu TS, Beek AM, Hofman MB, Heusen NS, van Rossum AC. Myocardial viability: rapid assessment with delayed contrast-enhanced MR imaging with three-dimensional inversion-recovery prepared pulse sequence. Radiology 2004;230(2):576-82.

[11] Fieno DS, Kim RJ, Chen EL, Lomasney JW, Klocke FJ, Judd RM. Contrast-enhanced magnetic resonance imaging of myocardium at risk: distinction between reversible and irreversible injury throughout infarct healing. J Am Coll Cardiol 2000;36(6):1985-91.

[12] Amado LC, Gerber BL, Gupta SN, Rettmann DW, Szarf G, Schock R, et al. Accurate and objective infarct sizing by contrast-enhanced magnetic resonance imaging in a canine myocardial infarction model. J Am Coll Cardiol 2004;44(12):2383-9.

[13] Schuijf JD, Kaandorp TA, Lamb HJ, van der Geest RJ, Viergever EP, van der Wall EE, et al. Quantification of myocardial infarct size and transmurality by contrastenhanced magnetic resonance imaging in men. Am J Cardiol 2004;94(3):284-8.

[14] Cerqueira MD, Weissman NJ, Dilsizian V, Jacobs AK, Kaul S, Laskey WK, et al. Standardized myocardial segmentation and nomenclature for tomographic imaging of the heart: a statement for healthcare professionals from the Cardiac Imaging Committee of the Council on Clinical Cardiology of the American Heart Association. J Nucl Cardiol 2002;9(2):240-5.

Address for correspondence

Nadjia Kachenoura

INSERM U678, 91 bvd de l'Hôpital, F-75634 Paris cedex 13 France

E-mail address kacheno@imed.jussieu.fr 